\title{
WILLMORE LAGRANGIAN SUBMANIFOLDS IN COMPLEX PROJECTIVE SPACE
}

\author{
SHICHANG SHU AND SANYANG LIU
}

Abstract. Let $M$ be an $n$-dimensional compact Willmore Lagrangian submanifold in a complex projective space $C P^{n}$ and let $S$ and $H$ be the squared norm of the second fundamental form and the mean curvature of $M$. Denote by $\rho^{2}=S-n H^{2}$ the non-negative function on $M, K$ and $Q$ the functions which assign to each point of $M$ the infimum of the sectional curvature and Ricci curvature at the point. We prove some integral inequalities of Simons' type for $n$-dimensional compact Willmore Lagrangian submanifolds in $C P^{n}$ in terms of $\rho^{2}, K, Q$ and $H$ and obtain some characterization theorems.

Mathematics subject classification (2010): 53C42, 53C40.

Keywords and phrases: Willmore Lagrangian submanifolds; complex projective space; curvature; totally umbilical.

\section{REFERENCES}

[1] B. Y. CHEn, Some conformal invariants of submanifolds and their applications, Boll. Un. Mat. Ital., 10 (1974), 380-385.

[2] B. Y. Chen And K. Ogiue, On totally real submanifolds, Trans. Amer. Math. Soc., 193 (1974), 257-266.

[3] Q.-M. Cheng, Submanifolds with constant scalar curvature, Proc. of the Royal Soc. of Edinburgh, 132A (2002), 1163-1183.

[4] S. Y. Cheng And S. T. YAU, Hypersurfaces with constant scalar curvature, Math. Ann., 225 (1977), 195-204.

[5] S. S. Chern, M. Do CARmo And S. Kobayashi, Minimal submanifolds of a sphere with second fundamental form of constant length, in Functional Analysis and Related Fields (F. Brower, ed.), Springer-Verlag, Berlin, 1970, 59-75.

[6] Z. Guo, H. LI AND C. P. WANG, The second variation formula for Willmore submanifolds in $S^{n}$, Results in Math., 40 (2001), 205-225.

[7] R. Harvey and H. B. Lawson, Calibrated geometries, Acta Math., 148 (1982), 47-157.

[8] Z. HU AND H. LI, Willmore submanifolds in a Riemannian manifold, Proceedings of the workshop Contemporary Geometry and Related Topics, World Scientific, September 15, 2003, 275-299.

[9] Z. HU, AND H. Li, Willmore Lagrangian spheres in the complex Euclidean space $C^{n}$, Ann. of Global of Anal. and Geom., 25 (2004), 73-98.

[10] A. M. Li AND G. ZHAO, Totally real minimal submanifolds in $C P^{n}$, Arch. Math., 62 (1994), 562-568.

[11] H. LI, Willmore submanifolds in a sphere, Math. Res. Letters, 9 (2002), 771-790.

[12] H. Li AND L. VRANCKEN, A basic inequality and new characterization of Whitney spheres in a complex space form, Israel J. of Math., 146 (2005), 223-242.

[13] G. D. Ludden, M. OKumura AND K. Yano, A totally real surface in $C P^{2}$ that is not totally geodesic, Proc. of Amer. Math. Soc., 53 (1975), 186-190.

[14] Y. G. OH, Second variation and stabilities of minimal Lagrangian submanifolds in Kähler manifolds, Invent. Math., 101 (1990), 501-519.

[15] F. J. Pedit And T. J. Willmore, Conformal geometry, Atti Sem. Mat. Fis, Univ Modena, XXXVI (1988), 237-245. 
[16] Y. SHEN, $N$-dimensional Minimal Totally real submanifolds in a complex projective space $C P^{n}$, Advance in Math. (Chinese), 13 (1984), 65-70.

[17] A. Strominger, S. T. YAU and E. Zaslow, Mirror symmetry is T-duality, Nuclear Physics, B479 (1996), 243-259.

[18] C. P. WANG, Moebius geometry of submanifolds in $S^{n}$, Manuscripta Math, 96 (1998), 517-534. 